www.nature.com/pj

\title{
Denaturant-induced helix-coil transition of oligopeptides: theoretical and equilibrium studies of short oligopeptides C17 and AK16
}

\author{
Fumiaki Kanô ${ }^{1,5}$, Masaji Shinjo ${ }^{2,5}$, Zhi-jie Qin ${ }^{2,6}$, Jinsong Li $^{2}$, Yoshitaka Matsumura ${ }^{2}$, Akio Shimizu ${ }^{3}$, \\ Akio Teramoto $^{4,4}$ and Hiroshi Kihara ${ }^{2}$
}

A statistical mechanical theory on the effects of denaturant on the helix-coil transition of polypeptides was developed. In the proposed theory, unfolding agents were assumed to interact with the polypeptide backbone. The theoretical results were compared with the findings of guanidine-induced helix-coil transition experiments, which were conducted on short peptides (C17 and AK16). Specifically, the helix fraction and average number of helices in C17 and AK16 were estimated. Under unfolding conditions (high denaturant concentration), the number of helices in a given sequence was close to zero. The radius of gyration was measured, and the results were related to those of the proposed theory.

Polymer Journal (2011) 43, 293-300; doi:10.1038/pj.2010.143; published online 2 February 2011

Keywords: AK16; C17; helix-coil transition; $\alpha$-helix; protein denaturation; unfolding by denaturant; Zimm-Bragg theory

\section{INTRODUCTION}

The $\alpha$-helix is an important structural unit that is commonly found in proteins. $\alpha$-Helices can transform into the coiled state based on the temperature, $\mathrm{pH}$ and the concentration of denaturants, such as urea and guanidium salts. Helix-coil transitions of polypeptides have been extensively studied by Zimm and Bragg, ${ }^{1}$ Nagai $^{2}$ and Lifson and Roig ${ }^{3}$ (see for reviews ${ }^{4,5}$ ).

In this study, we developed a denaturant-induced helix-coil transition theory based on statistical physics. Denaturant-induced protein unfolding was initially studied by Tanford et al. (see for reviews ref. ${ }^{6,7}$ ) and has been extensively investigated by many other researchers (see for reviews ref. ${ }^{8,9}$ ). However, the mechanism of unfolding has not yet been elucidated. One possible mechanism of denaturant-induced protein unfolding involves a change in the solvent properties of water. These effects could alter the packing interactions of $\alpha$-helices and $\beta$-sheets. Another possible mechanism may include specific interactions with functional groups on the protein. In fact, the interaction of denaturants with nonpolar and polar surfaces is more favorable than that of water. Interactions between the denaturant and the peptide backbone disturb hydrogen bonds in water and the interior of the protein. Thus, to understand the unfolding of $\alpha$-helices in proteins by denaturants, interactions between the denaturant and the peptide backbone must be understood.

Schellman ${ }^{10,11}$ developed a protein unfolding theory based on the selective solvation of denaturants thermodynamically. Using
Schellman's theory, the effects of denaturants on helix-coil transitions have been studied by Scholtz et al.,12 who connected the thermodynamic solvation model derived by Schellman ${ }^{10,11}$ to the Zimm and Bragg model for the analysis of the unfolding of peptide helices by urea. However, the method was phenomenological and was not consistently based on statistical physics.

For the helix-coil transition, two types of theory based on the transfer-matrix method have been used to explain the molecular mechanism of these phenomena. Hydrogen bonds were evaluated in one theory, ${ }^{1}$ and the other theory considered residue conformations. ${ }^{3}$ $\mathrm{Nagai}^{2}$ developed these treatments into a new theory based on the spatial conformation of the helix.

In this study, we developed a new denaturant-induced helix-coil transition theory through a propagator method. The proposed theory is easy to handle, and the physical averaged quantities are simple to calculate ${ }^{13-15}$ Moreover, the effect of denaturants on the statistical weight was considered. The grand partition function was calculated, and averaged quantities, such as the helix fraction, number of helices and end-to-end distance of the polypeptide, were derived as a function of the denaturant concentration.

We analyzed the guanidine hydrochloride ( $\mathrm{GuHCl})$-induced helix-coil transition of short peptides (C17 and AK16) according to the proposed theory. C17 consists of DLTDDIMCVKKILDKVG, corresponding to $\alpha$-helical region of the 84 th to 100 th amino acid

\footnotetext{
${ }^{1}$ Department of Physics, Showa University, 4562 Kamiyoshida, Fujiyoshida, Japan; ${ }^{2}$ Department of Physics, Kansai Medical University, Hirakata, Japan; ${ }^{3}$ Department of Environmental Engineering, Symbiosis Faculty of Engineering, Soka University, Hachioji, Japan and ${ }^{4}$ Faculty of Science and Engineering, Ritsumeikan University, Shiga, Japan 5These two authors contributed equally to this work.

${ }^{6}$ Current address: Department of Chemistry and Biochemistry, University of California at Santa Cruz, Santa Cruz, CA 95064, USA.

*This paper is dedicated to Akio Teramoto, who passed away on 7 February 2010.

Correspondence: Professor H Kihara, Department of Physics, Kansai Medical University, 18-89 Uyama-Higashi, Hirakata 573-1136, Japan,

E-mail: kihara@makino.kmu.ac.jp
}

Received 17 August 2010; revised 10 November 2010; accepted 22 November 2010; published online 2 February 2011 
of bovine $\alpha$-lactalbumin. Alternatively, AK16 consists of Ac-YGAAKAAAAKAAAAKA-NH2.

\section{THEORY}

Statistical analysis of helix-coil transitions induced by denaturant Treatment by $\mathrm{Doi}^{14}$ can be extended to include the effects of denaturation by denaturants. We assumed that the denaturant molecules only bind to the coil of the polypeptide and that the surface of the helix becomes unavailable for binding.

For each coiled portion of $n$ peptide units (residues), the statistical weight between the start point $r$ and the end point $r^{\prime}$ can be expressed as

$$
G_{c}\left(\boldsymbol{r}-\boldsymbol{r}^{\prime}, n, m\right)=\left(\begin{array}{c}
p n \\
m
\end{array}\right) q^{m} H_{c}\left(\boldsymbol{r}-\boldsymbol{r}^{\prime}, n\right)
$$

with

$$
H_{c}(\boldsymbol{r}, n)=\exp \left(-\frac{\Delta g}{k_{\mathrm{B}} T} n\right)\left(\frac{2 \pi n b^{2}}{3}\right)^{-3 / 2} \exp \left(-\frac{3 \boldsymbol{r}^{2}}{2 n b^{2}}\right)
$$

where $m$ is the number of residues that are attached to denaturant molecules, $p$ is the maximum number of attached denaturant molecules per residue, $q$ is the partition function, which is dependent on the internal degree of freedom of denaturant molecules attached to a residue, $b$ is the effective bond length of the residue, $\Delta g$ is the difference in the free energy of the coiled and helical portion of the residue (which implies that all free energies are measured from the complete helix state), $k_{\mathrm{B}}$ is the Boltzmann constant and $T$ is the absolute temperature.

For the helical portion of $n$ peptide units, the statistical weight between point $\boldsymbol{r}$ and point $\boldsymbol{r}^{\prime}$ can be expressed as

$$
G_{h}\left(\boldsymbol{r}-\boldsymbol{r}^{\prime}, n\right)=\frac{\delta\left(\left|\boldsymbol{r}-\boldsymbol{r}^{\prime}\right|-n a\right)}{4 \pi n^{2} a^{2}}
$$

where $a$ is the length of the bond projected onto the helical axis.

We assumed that both ends of the peptide were in the coiled state; thus, the statistical weight of the system, which consists of $N$ peptide units and end-to-end vector $\boldsymbol{R}$, can be written as

$$
\begin{aligned}
Q(\boldsymbol{R}, N, \lambda) & =\int d^{3} r_{1} d n_{1} \sum_{m_{1}=0}^{n_{1}}\left(\begin{array}{c}
p n_{1} \\
m_{1}
\end{array}\right)(q \lambda)^{m_{1}} H_{c}\left(r_{1}, n_{1}\right) \\
& +v^{2} \iint d^{3} \boldsymbol{r}_{1} d n_{1} d^{3} r_{2} d n_{2} \sum_{m_{1}=0}^{n_{1}}\left(\begin{array}{c}
p n_{1} \\
m_{1}
\end{array}\right)(q \lambda)^{m_{1}} H_{c}\left(\boldsymbol{r}_{1}, n_{1}\right) \\
& \times G_{h}\left(\boldsymbol{r}_{2}, n_{2}\right) \times \sum_{m_{3}=0}^{n_{3}}\left(\begin{array}{c}
p n_{3} \\
m_{3}
\end{array}\right)(q \lambda)^{m_{3}} H_{c}\left(\boldsymbol{r}_{3}, n_{3}\right) \\
& +\cdots=\sum_{i=0}^{\infty} v^{2 i} \int d^{3} \boldsymbol{r}_{1} d n_{1} \cdots \int d^{3} \boldsymbol{r}_{2 i} d n_{2 i}(1+q \lambda)^{p\left(n_{1}+n_{3}+\cdots+n_{2 i+1}\right)} Q_{1}
\end{aligned}
$$

with

$$
Q_{1}=H_{c}\left(\boldsymbol{r}_{1}, n_{1}\right) G_{h}\left(\boldsymbol{r}_{2}, n_{2}\right) \cdots H_{c}\left(\boldsymbol{r}_{2 i+1}, n_{2 i+1}\right)
$$

where $v$ is the statistical weight of the boundary between the random coil sequences and the helix, at which there is a lack of hydrogen bonding, and $\lambda$ is the absolute activity of the denaturant molecule. The value of the parameter $v$ was identical to that assigned to the helix termini by Lifson and Roig. ${ }^{3}$ Because $N$ is a large number, $n_{\mathrm{i}}$ is considered as a continuous number and can be integrated. All possible compositions must be counted under the conditions that the total number of peptide units is equal to $N$ and that the length of the endto-end vector is equal to $\boldsymbol{R}$. To avoid this complication, Laplace transforms and Fourier transforms were conducted on $N$ and $\boldsymbol{R}$, respectively.

$$
\begin{aligned}
\hat{Q}(\boldsymbol{k}, \omega, \lambda) & =\int_{0}^{\infty} d S e^{-\omega S} \int Q(\boldsymbol{R}, S, \lambda) e^{-i k R} d^{3} R \\
& =\frac{G_{c}(\boldsymbol{k}, \omega, \lambda)}{1-v^{2} G_{c}(\boldsymbol{k}, \omega, \lambda) G_{h}(\boldsymbol{k}, \omega)}
\end{aligned}
$$

with

$$
\begin{aligned}
& G_{c}(\boldsymbol{k}, \omega, \lambda)=\left(\omega+\epsilon+\frac{b^{2} k^{2}}{6}\right)^{-1} \\
& G_{h}(\boldsymbol{k}, \omega)=-\frac{1}{2 i k a} \ln \frac{i \omega+k a}{i \omega-k a} \\
& \epsilon=\epsilon_{0}-p \ln (1+q \lambda) \\
& \epsilon_{0}=\frac{\Delta g}{k_{B} T} \\
& \lambda=\frac{\beta}{q_{s}} C^{\prime}, \quad \beta=\frac{M_{w}}{10^{3} \gamma M_{s}}
\end{aligned}
$$

$\epsilon$ is the reduced free energy difference between the coiled and helix portion of the residue, and $\epsilon_{0}$ is the $\epsilon$-value of the residue in the absence of the denaturant. $q_{\mathrm{s}}$ is the partition function originating from the internal degree of freedom of the denaturant molecule in solution, $M_{\mathrm{w}}$ and $M_{\mathrm{s}}$ are the molecular weight of water and the denaturant, respectively, and $\gamma\left(\mathrm{gl}^{-1}\right)$ and $C^{\prime}\left(\mathrm{mgl}^{-1}\right)$ are the density of the solution and the concentration of solute, respectively. ${ }^{16}$

Thus, the statistical weight of a system composed of $N$ peptide units can be obtained by reverse transforming Equation (6).

$$
\begin{aligned}
\hat{\hat{Q}}(\boldsymbol{k}, N, \lambda)= & \frac{1}{2 \pi i} \int_{d-i \infty}^{d+i \infty} e^{\omega N} \hat{Q}(\boldsymbol{k}, \omega, \lambda) d \omega \\
& =\int Q(\boldsymbol{R}, N, \lambda) e^{-i \boldsymbol{k}} d^{3} R \\
& =\Xi(N, \lambda)\left(1-\frac{k^{2}}{6}<R^{2}(N, \lambda)>+\cdot \cdot\right)
\end{aligned}
$$

where $d$ is a real number that is larger than the real part in any pole of $\hat{Q}(\boldsymbol{k}, \omega, \lambda)$ in the $\omega$ plane. The grand partition function $\Xi(N, \lambda)$ of a system composed of $N$ units can be expressed as

$$
\begin{aligned}
\Xi(N, \lambda)= & \hat{\hat{Q}}(\boldsymbol{k}=0, N, \lambda) \\
& =\frac{1}{2 \pi i} \oint_{C} e^{\omega N} \hat{Q}(\boldsymbol{k}=\boldsymbol{0}, \omega, \lambda) d \omega
\end{aligned}
$$

The integral in Equation (12) transforms into the contour integral, including the poles. The contour of the integral of $C$ is shown in Figure 1:

$$
\Xi(N, \lambda)=\frac{\omega_{+} e^{\omega_{+} N}-\omega_{-} e^{\omega_{-} N}}{\omega_{+}-\omega_{-}}
$$

with

$$
\omega_{ \pm}=\frac{-\epsilon \pm \sqrt{\epsilon^{2}+4 v^{2}}}{2}
$$

Our parameters correspond approximately to the Zimm-Bragg parameters and the Lifson-Roig parameters: ${ }^{1,3,4}$

$$
e^{\epsilon}=s=\frac{w}{1+v}
$$




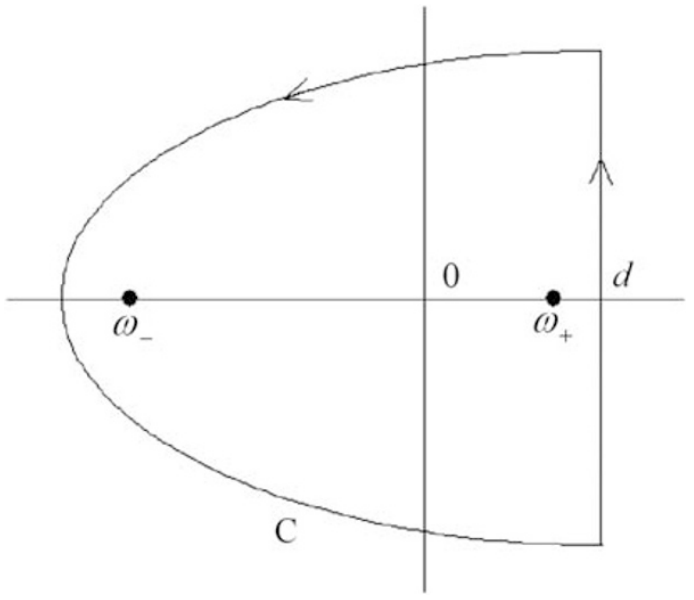

Figure 1 Graphical representation of the contour of integral $C$ of Equation (13).

$$
\sigma=\frac{v^{2}}{(1+v)^{4}}
$$

where $s$ and $\sigma$ are the helix propagation parameter and the helix nucleation parameter, respectively, and $w$ is a parameter of the LifsonRoig model and is a weight assigned to helical residues in the interior of the helix.

We refer to the quantity $q \lambda$ as the denaturant molarity. According to its definition, we can transform Equation (11) into the following expression:

$$
q \lambda=\eta C
$$

where $C$ is the denaturant molarity and $\eta$ is the coefficient of $C$, which can be expressed as

$$
\begin{aligned}
& C=\frac{C^{\prime}}{10^{3} M s} \\
& \eta=\frac{M_{W}}{\gamma} \frac{q}{q_{s}}
\end{aligned}
$$

The dependence of the helical structure on the molarity of the denaturant can be used to quantify the effects of the denaturant on the unfolding of the helix. The reduced free energy, $\epsilon$, was calculated according to Equation (9) and is plotted in Figure 2 as a function of the denaturant molarity at various values of $p$. The value of $\epsilon$ in the transition range decreased almost linearly as the value of $C$ increased.

\section{Average quantities}

Ratio of peptides in the helix state: $f_{\mathrm{H}}$. Based on Equation $(14), f_{\mathrm{H}}$ was obtained from the following equation:

$$
\begin{aligned}
f_{\mathrm{H}}= & 1+\frac{1}{N} \frac{\partial \ln \Xi}{\partial \epsilon} \\
& =\frac{1}{2}\left(1+\frac{\epsilon}{\sqrt{\epsilon^{2}+4 v^{2}}}\right)+\frac{1}{2 N A} \times\left[-1+e^{-\left(\omega_{+}-\omega_{-}\right) N}\right. \\
& +\left\{1+\left(1+2 N \omega_{-}\right) e^{-\left(\omega_{+}-\omega_{-}\right) N}\right\} \\
& \left.\times \frac{\epsilon}{\sqrt{\epsilon^{2}+4 v^{2}}}\right]-\frac{\epsilon}{N\left(\epsilon^{2}+4 v^{2}\right)}
\end{aligned}
$$

with

$$
A=\omega_{+}-\omega_{-} \times e^{-\left(\omega_{+}-\omega_{-}\right) N}
$$

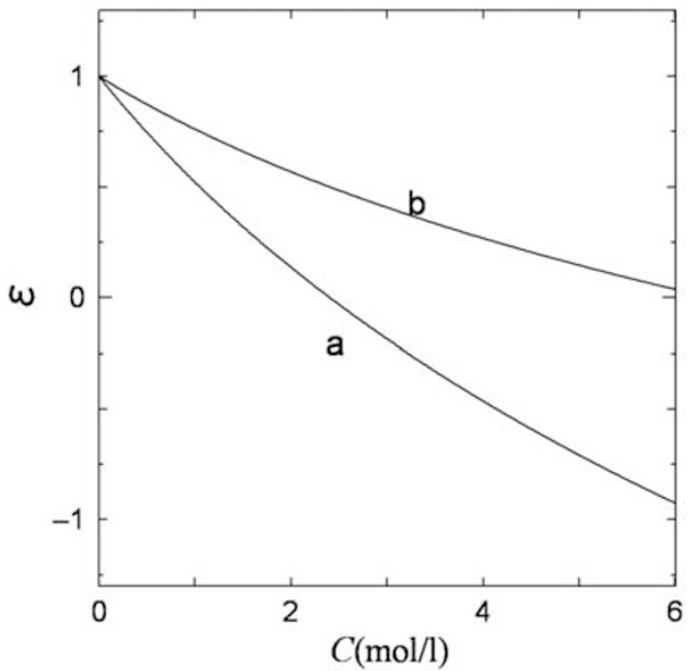

Figure 2 Dependence of the reduced free energy, $\epsilon$, on the denaturant molarity. The numerical values were $\epsilon_{0}=1.0$ and $\eta=0.27$. For a and $\mathrm{b}, p=2$ and 1 , respectively.

Average number of units in the helix state: $\boldsymbol{i}_{\mathrm{H}}$. Based on Equation (14), $i_{\mathrm{H}}$ was obtained from the following equation:

$$
\begin{aligned}
i_{\mathrm{H}}= & \frac{\partial \ln \Xi}{\partial \ln v^{2}}=\frac{v^{2}}{\sqrt{\epsilon^{2}+4 v^{2}}} \\
& \times\left[N+\frac{1}{A}\left\{1+\left(1+2 N \omega_{-}\right) e^{-\left(\omega_{+}-\omega_{-}\right) N}\right\}-\frac{2}{\sqrt{\epsilon^{2}+4 v^{2}}}\right]
\end{aligned}
$$

Mean square end-to-end distance of a peptide: $\left\langle R^{2}\right\rangle$. Based on Equation (14), $\left\langle R^{2}\right\rangle$ was obtained from the following equation:

$$
\begin{aligned}
\left\langle R(N)^{2}\right\rangle= & -\frac{6}{\Xi(N, \lambda)} \lim _{k \rightarrow 0} \frac{\partial Q(\boldsymbol{k}, N, \lambda)}{\partial k^{2}} \\
= & -\frac{6}{\Xi(N, \lambda)} \times \frac{1}{2 \pi i} \int_{d-i \infty}^{d+i \infty} e^{\omega N} \frac{b^{2} \omega^{3}+2 v^{2} a^{2}}{\omega\left(\omega^{2}+\epsilon-v^{2}\right)} d \omega \\
= & -\frac{6}{\Xi(N, \lambda)}\left\{\left(B+D N+B N \omega_{+}\right) e^{\omega_{+} N}\right. \\
& \left.+\left(E+F N+E N \omega_{-}\right) N e^{\omega_{-} N}+\frac{G}{6}\right\}
\end{aligned}
$$

where

$$
\begin{aligned}
& B=\frac{\omega_{+} \omega_{-}^{2} G+4 \omega_{-}\left(\omega_{+}-\omega_{-}\right) D}{2 \omega_{+}\left(\omega_{-}^{2}-\omega_{+}^{2}\right)} \\
& D=\frac{\frac{\epsilon b^{2}}{2}+\left(2 \omega_{-}^{2}-\frac{\omega_{-}^{3}}{\omega_{+}}\right) G}{3\left(\omega_{+}-\omega_{-}\right)^{3}} \omega_{+}^{2} \\
& E=-B-\frac{G}{6} \\
& F=-\frac{2 a^{2} \epsilon}{3 \omega_{+}^{2}}-\left(\frac{\omega_{-}}{\omega_{+}}\right)^{2} D \\
& G=-2\left(\frac{a}{v}\right)^{2}
\end{aligned}
$$




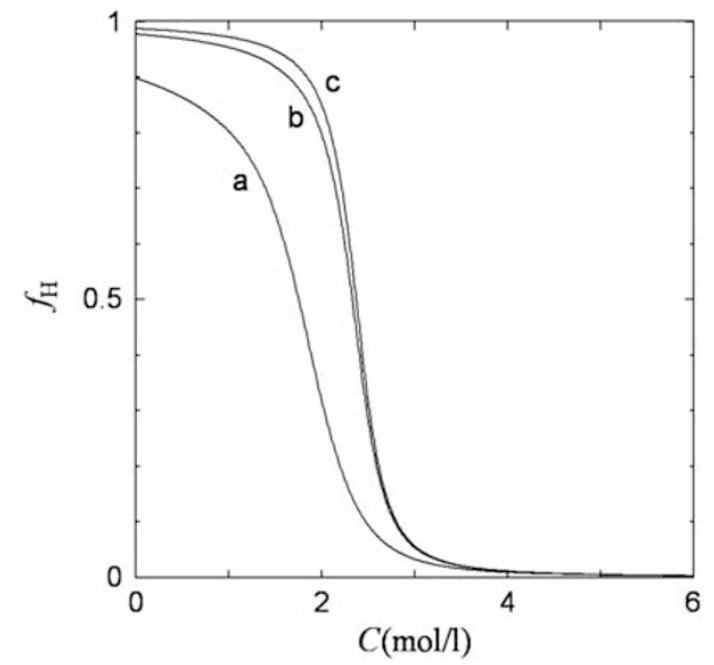

Figure 3 The fraction of the helix state, $f_{\mathrm{H}}$, as a function of the denaturant molarity, $C$. The curves were drawn according to Equation (21). The numerical values were $v=0.05, \epsilon_{0}=1.0, \eta=0.27$ and $p=2$. $N=20$ for a, $N=100$ for $\mathrm{b}$ and $N=200$ for $\mathrm{c}$.

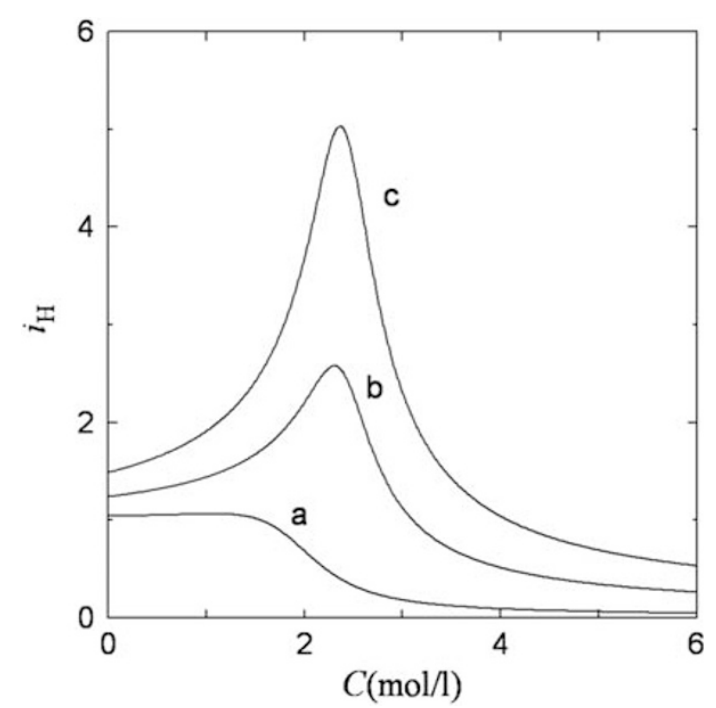

Figure 4 The number of helices as a function of $C$. The curves were drawn according to Equation (23). The numerical values of the parameters are identical to those in Figure 3.

In Figures 3-5, the following quantities are shown as a function of the solvent molarity $(C)$ : the ratio of peptide in the helix state (Figure 3 ), the average number of helix (Figure 4) and the root mean square end-to-end distance divided by $\sqrt{N b^{2}}, \xi=\sqrt{\left\langle R(N)^{2}\right\rangle / N b^{2}}$ (Figure 5). The results shown in the figures clearly demonstrate that the helix content and the average number of helix change near the transition point, $\epsilon \sim 0$. The transition becomes sharper as the number of residues increases. This property is closely related to the fact that a minimum is observed when the radius of gyration is plotted as a function of solution condition. ${ }^{17}$ In a short peptide, there is zero or one helix through out the transition process, as shown in Figure 4. The helix moves forward or backward due to the winding and unwinding process at both ends of the chain ${ }^{18}$ and is attacked by denaturant molecules.

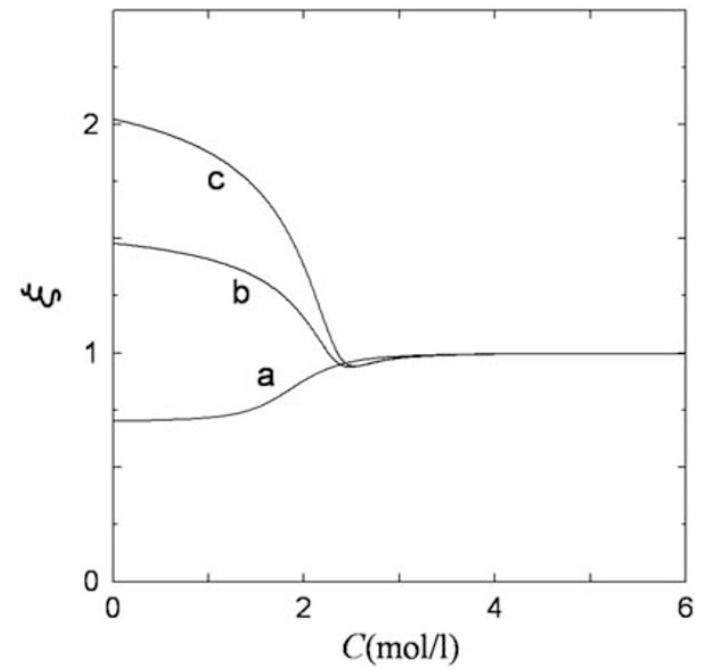

Figure 5 The value of $\xi$, the root mean square end-to-end distance divided by $\sqrt{N b^{2}}$ as a function of $C$, according to Equation (24). The numerical value of $b / a=10$. The values of the other parameters are identical to those shown in Figure 3.

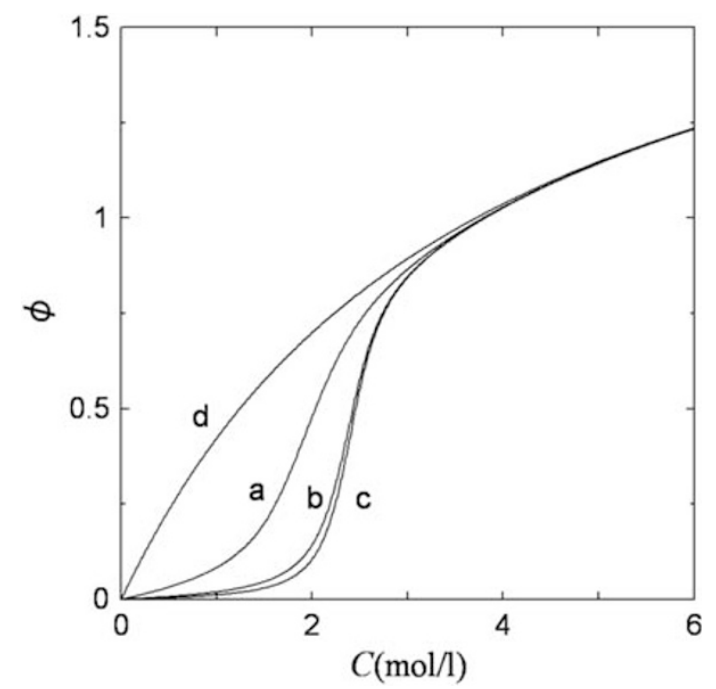

Figure 6 Dependence of $\phi$ on the denaturant molarity. The curves were drawn according to Equation (30). The numerical values for the parameters are identical to those shown in Figure 3. The other numerical values for $d$ are $v=0.05, \epsilon_{0}=-0.10, \eta=0.27, p=2$ and $N=20$.

Degree of denaturant binding: $\boldsymbol{\phi}$. The ratio of the number of denaturant molecules bound to a residue can be expressed as

$$
\phi=\frac{1}{N} \frac{\partial \ln \Xi}{\partial \ln \lambda}=\frac{p q \lambda}{1+q \lambda}\left(1-f_{\mathrm{H}}\right)
$$

The fraction $1-f_{\mathrm{H}}$ is the degree of residues in the random coiled state. Although the formation of intramolecular hydrogen bonds is a cooperative phenomena, the degree of denaturant binding is given as the product of the coil fraction $\left(1-f_{\mathrm{H}}\right)$ and the coverage of the Langmuir isotherm. The characteristics of $\phi$ in Equation (30) were nearly identical to the results obtained by Bixon and Lifson. ${ }^{19}$ In Figure 6, notable features of the system are revealed. For instance, when the initial state of the peptide is helical $\left(\epsilon_{0}>0\right)$, the degree of denaturant binding $(\phi)$ produces a sigmoidal curve. Under these conditions, $\phi$ can be attributed to the coil fraction, $1-f_{\mathrm{H}}$. Alternatively, 
when the initial state of the peptide is coiled $\left(\epsilon_{0}<0\right), \phi$ demonstrates Langmuir-type binding.

Helix fraction and number of the Lifson-Roig model If $v^{2}$ is small, Equation (17) can be approximated as

$$
v^{2}=\sigma
$$

In this case, $f_{\mathrm{H}}$ and $i_{\mathrm{H}}$ can be approximated as

$$
\begin{aligned}
f_{\mathrm{H}}= & f 1-\frac{2 \sqrt{f(1-f)}}{N \sigma^{1 / 2}}+\left[1+\frac{2 \sqrt{f(1-f)}}{N \sigma^{1 / 2}}\right] \\
& \left.\times \exp \left[-\frac{N \sigma^{1 / 2}}{\sqrt{f(1-f)}}\right]\right\} \\
& \times\left\{1+\left(\frac{f}{1-f}\right) \exp \left[-\frac{N \sigma^{1 / 2}}{\sqrt{f(1-f)}}\right]\right\}^{-1} \\
= & f(1-f)\left\{1-\frac{2}{N P}+\left(1+\frac{2}{N P}\right) e^{-N P}\right\} \\
& \times\left(1-f+f e^{-N P}\right)^{-1}
\end{aligned}
$$

and

$$
\begin{aligned}
i_{\mathrm{H}}= & f(1-f)\left(\frac{N \sigma^{1 / 2}}{\sqrt{f(1-f)}}-2\right) \\
& +f\left\{1+\left(1-\frac{2 N f \sigma^{1 / 2}}{\sqrt{f(1-f)}}\right) \exp \left[-\frac{N \sigma^{1 / 2}}{\sqrt{f(1-f)}}\right]\right\} \\
& \times\left\{1+\left(\frac{f}{1-f}\right) \exp \left[-\frac{N \sigma^{1 / 2}}{\sqrt{f(1-f)}}\right]\right\}^{-1} \\
= & f(1-f)\left[N P-2+\left\{1+(1-2 f N P) e^{-N P}\right\}\right. \\
& \left.\times\left(1-f+f e^{-N P}\right)^{-1}\right]
\end{aligned}
$$

where

$$
f=\frac{1}{2}\left(1+\frac{\epsilon}{\sqrt{\epsilon^{2}+4 v^{2}}}\right)=\frac{1}{2}\left[1+\frac{\ln s}{\sqrt{(\ln s)^{2}+4 \sigma}}\right]
$$

and

$$
P=\frac{\sigma^{1 / 2}}{\sqrt{f(1-f)}}
$$

Equation (32) is equal to the equation obtained by Teramoto. ${ }^{20}$

\section{EXPERIMENTAL PROCEDURE}

Circular dichroism (CD) was conducted on an UNISOKU (Unisoku Inc., Hirakata, Osaka, Japan) spectropolarimeter, and the C17 and AK16 peptides were synthesized according to standard procedure (AS).

For the $\mathrm{CD}$ measurements, $\mathrm{C} 17$ and AK16 peptides were dissolved in a mixture of $0.01 \mathrm{M}$ phosphate buffer (30\%) and methanol (70\%). For C17 and AK16, the $\mathrm{pH}$ of the buffer was set to 2.0 and 7.0, respectively, and the measurements were conducted at $-40{ }^{\circ} \mathrm{C}$.

$\mathrm{X}$-ray scattering experiments were performed on $\mathrm{C} 17$ at the beam line 15A small angle installation (BL-15A) of the Photon Factory at High Energy Acceleration Research Organization (KEK), Tsukuba, Japan. A stable beam of photons with a wavelength of $1.50 \AA$ was provided by a horizontally focusing bent-crystal monochromator and a vertically focusing mirror. ${ }^{21}$ Scattering data were obtained with a CCD-based X-ray detector (Hamamatsu Photonics K.K., Hamamatsu, Japan) ${ }^{22}$ and corrected for image distortion, nonuniform sensitivity and contrast reduction with an X-ray image intensifier before the analysis. ${ }^{23,24}$ The detector was set at a distance of $1000 \pm 10 \mathrm{~mm}$ from the sample. The concentration of peptides was set to $1 \mathrm{mg} \mathrm{ml}^{-1}$, and the temperature was maintained at $25^{\circ} \mathrm{C}$. A $0.01 \mathrm{M}$ phosphate buffer in $70 \%$ methanol at $\mathrm{pH} 2.0$ was used.

\section{ANALYSIS}

According to the proposed theory, $f_{\mathrm{H}}$ can be expressed as Equation (21). The parameter $\epsilon$ was converted into $s$ by Equation (16), and $s$ was related to the denaturant concentration $C$ :

$$
\frac{1}{s}=\frac{(1+\eta C)^{p}}{s_{0}}
$$

where

$$
s_{0}=\exp \left(\frac{\Delta g}{k_{\mathrm{B}} T}\right)
$$

Thus, $\epsilon$ and $C$ are directly related through $s$, and $f_{\mathrm{H}}$ can be expressed as a function of $C$.

$f_{\mathrm{H}}$ can be obtained from $\mathrm{CD}$ observations by using the following expression:

$$
f_{\mathrm{H}}=\frac{\theta-\theta_{\mathrm{C}}}{\theta_{\mathrm{H}}-\theta_{\mathrm{C}}}
$$

where $\theta$ is the mean residue ellipticity at $222 \mathrm{~nm}$ as a function of $C$, and $\theta_{\mathrm{C}}$ and $\theta_{\mathrm{H}}$ are the $\theta$-values of the coiled and helix state, respectively. $\theta_{\mathrm{C}}$ and $\theta_{\mathrm{H}}$ are also dependent on the denaturant concentration: ${ }^{25}$

$$
\begin{aligned}
& \theta_{\mathrm{C}}=\theta_{\mathrm{C} 0}+d_{\mathrm{c}} C \\
& \theta_{\mathrm{H}}=\theta_{\mathrm{H} 0}+d_{\mathrm{h}} C
\end{aligned}
$$

where $\theta_{\mathrm{C} 0}$ and $\theta_{\mathrm{H} 0}$ are the values of $\theta_{\mathrm{C}}$ and $\theta_{\mathrm{H}}$ at a denaturant concentration of $0 \mathrm{M}$, respectively. $d_{\mathrm{c}}$ and $d_{\mathrm{h}}$ are constants.

The $\theta$-values were fitted to the $\mathrm{CD}$ data by parameter fitting.

In the fitting analysis, $\theta_{\mathrm{C} 0}$ was set to $640\left(\mathrm{deg} \mathrm{cm}^{-2} \mathrm{dmol}^{-1}\right)^{26}$ for both peptides. $\theta_{\mathrm{H} 0}$ was estimated from the fitting calculation, along with $s_{0}$ and $\eta$. $d_{\mathrm{c}}$ and $d_{\mathrm{h}}$ were estimated from the experimental data presented in the Results section.

A two-state equilibrium was also considered:

$$
[\mathrm{N}]+m^{\prime}[\mathrm{D}] \leftrightharpoons\left[\mathrm{U}-m^{\prime} \mathrm{D}\right] K=\frac{\left[\mathrm{U}-m^{\prime} \mathrm{D}\right]}{[\mathrm{N}][\mathrm{D}]^{m^{\prime}}}
$$

where $[N]$ is the concentration of the peptide in the native state, $[D]$ is the concentration of the denaturant, $m^{\prime}$ is the number of bound denaturant molecules to an unfolded oligopeptide, [U- $\left.m^{\prime} \mathrm{D}\right]$ is the concentration of unfolded denaturant-bound oligopeptide and $K$ is the equilibrium constant.

For this reaction, $L_{\theta}$ was expressed according to the following equation:

$$
L_{\theta}=\frac{L_{\mathrm{H}}+K[\mathrm{D}]^{m^{\prime}} L_{\mathrm{U}}}{1+K[\mathrm{D}]^{m^{\prime}}}
$$

where $L_{\mathrm{H}}$ and $L_{\mathrm{U}}$ are the $\mathrm{CD}$ values of the native and denatured states, respectively.

\section{RESULTS}

The $\mathrm{CD}$ experiments were conducted on $\mathrm{C} 17$ and AK16 peptides, and the results are shown in Figures 7a and b. As shown in the figures, C17 and AK16 are present in the random coil form at high $\mathrm{GuHCl}$ concentrations. The $\theta_{222}$ values of the native and coiled states were linearly proportional to the $\mathrm{GuHCl}$ concentration. The transition curve was fitted to Equation (41), as shown in Figures $7 \mathrm{a}$ and b. For C17, $K=0.04$ and $m^{\prime}=5.3$. Alternatively, for AK16, $K=0.0004$ and $m^{\prime}=7.6$. The experimental data shown in Figures $7 \mathrm{a}$ and $\mathrm{b}$ were also 

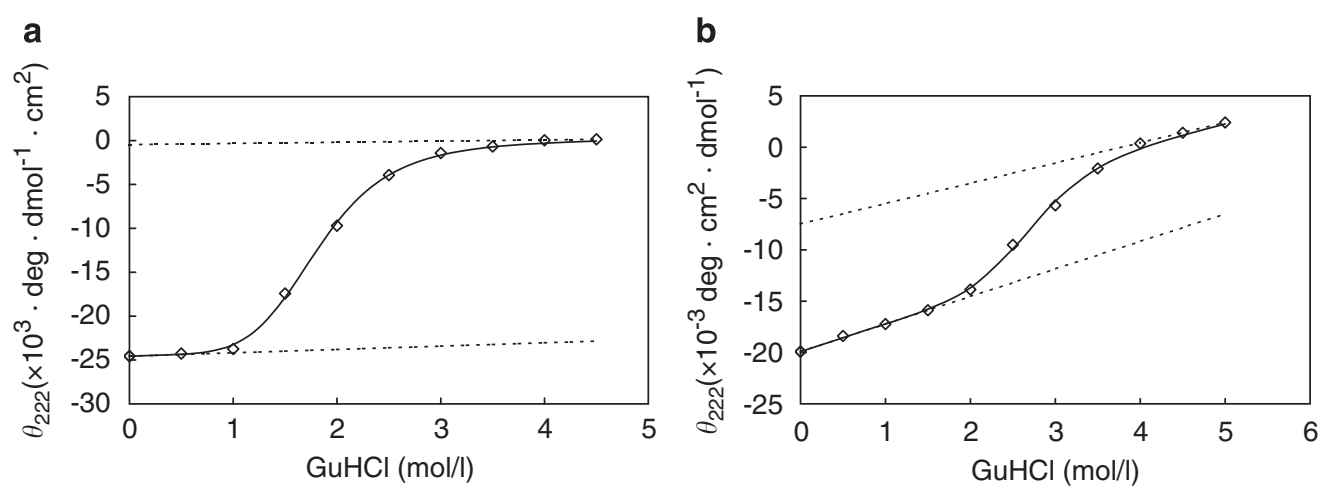

Figure 7 The experimental circular dichroism results. $\theta_{222}$ versus the guanidine hydrochloride $(\mathrm{GuHCl})$ concentration. The solid line represents the fit of Equation (41). The measurements were conducted at $-40{ }^{\circ} \mathrm{C}$ in the presence of $70 \%$ methanol at $\mathrm{pH} 2.0$ and 7.0 for $\mathrm{C} 17$ and $\mathrm{AK} 16$, respectively. (a) $\mathrm{C} 17$ and (b) AK16.

a

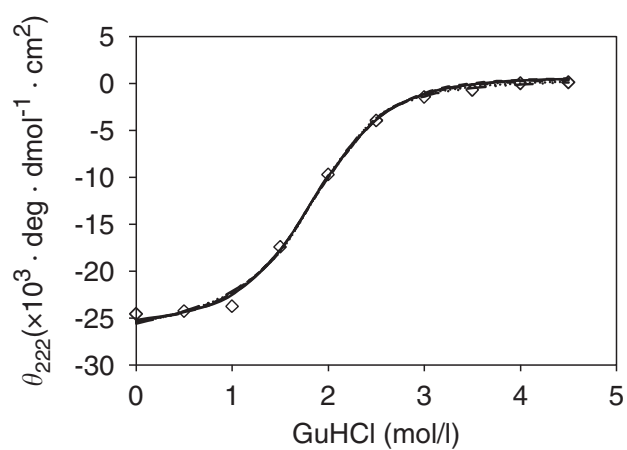

b

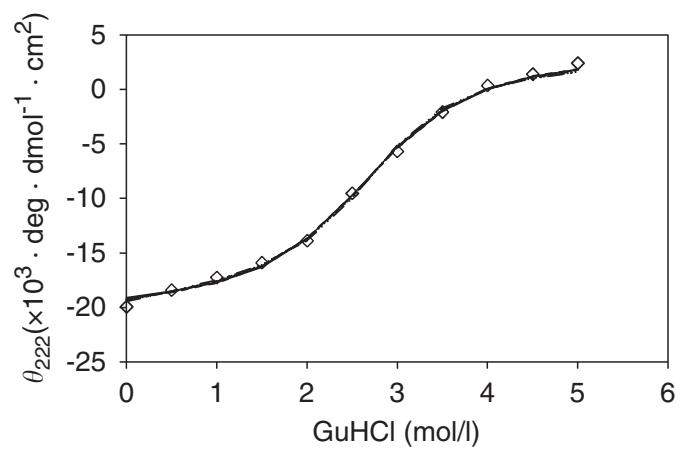

Figure 8 Experimental circular dichroism results. The fitted lines were obtained from statistical theory (Equations $16,21,36$ and 38 ). (a) C17 and (b) AK16. In both figures, six fitted lines were drawn at $v^{2}=10^{-2}, 10^{-3}$ and $10^{-4}$, and $p=1$ and 2 . The six fitted lines nearly overlap.

simulated according to statistical theory (Equations 16, 21, 36 and 38). According to Equation (38), $\theta$ is considered a function of $f_{\mathrm{H}}$, and $f_{\mathrm{H}}$ is a function of $C$ through $\epsilon$ and $s$, as described in the Analysis section. For C17, we adopted $d_{\mathrm{c}}=d_{\mathrm{h}}=0$ because the data were nearly constant at high and low concentrations of denaturant. For AK16, we used $d_{\mathrm{c}}=d_{\mathrm{h}}=0.354 ; d_{\mathrm{c}}$ was estimated from two data points, $\theta_{\mathrm{C} 0}$ and $\theta$ value at a denaturant concentration of $5 \mathrm{M}$, and the same value was also used for $d_{\mathrm{h}}$. The results shown in Figures $8 \mathrm{a}$ and $\mathrm{b}$ were obtained from the fitting curves and were calculated at various $v^{2}$ and $p$-values. For all of the $p$ - and $v^{2}$-values, the model provided a satisfactory fit to the experimental data. $p$ is the number of denaturant-attachable sites per coiled residue and was assumed to be equal to 1 or 2 , based on the chemical structure of the peptide residue. Subsequently, the experimental and theoretical values of $1 / s$ were compared. Figure 9 shows a plot of $1 / s$ versus the $\mathrm{GuHCl}$ concentration at $v^{2}=10^{-4}$. To estimate the optimal $v^{2}$-value, the standard deviation $(\Delta)$ was calculated at various $v^{2}$-values:

$$
\Delta=\sqrt{\frac{\sum \Delta_{i}^{2}}{n^{\prime}}}
$$

where $\Delta_{i}$ is the difference between the fitted line and the experimental data at each concentration, and $n^{\prime}$ is the number of data points. In Figure 9 (inset), $\Delta$ is shown as a function of $v^{2}$. For AK16, $\Delta$ decreased and became nearly constant when $v^{2}$ was $<10^{-3}$. Moreover, for C17, $\Delta$ decreased when $v^{2}=10^{-4}$. On the basis of the results described in the literature, $10^{-3}$ to $10^{-4}$ are reasonable values for $v^{2,20,26,27}$ thus, $v^{2}=10^{-4}$ was used in subsequent analyses. The difference between $p=1$ and $p=2$ was not significant; thus, because similar results were obtained when $v^{2}=10^{-3}$, the data shown hereafter were obtained at $v^{2}=10^{-4}$. For C17, $s_{0}$ was 2.4-2.6, $\eta$ was $0.14-0.34$ and $\theta_{\mathrm{H} 0}$ was $-29000\left(\mathrm{deg} \mathrm{cm}^{-2} \mathrm{dmol}^{-1}\right)$ when $p=1$ and $p=2$. For AK16, $s_{0}$ was 2.6-2.8, $\eta$ was $0.09-0.26$ and $\theta_{\mathrm{H} 0}$ was $-22000\left(\mathrm{deg} \mathrm{cm}^{-2} \mathrm{dmol}^{-1}\right)$ when $p=1$ and $p=2$. In both cases, these $s_{0}$ values were greater than that $\left(s_{0}=1.39\right)$ reported by Scholtz et al. ${ }^{12}$

As shown in Figure 10, $f_{\mathrm{H}}$ was calculated from the fitted curve, and the experimental data shown in Figure 8 were plotted against $C$ for $\mathrm{C} 17$ and $\mathrm{AK} 16$. At a $\mathrm{GuHCl}$ concentration of $0 \mathrm{M}$, the proportion of the helix form of the peptide was $>85 \%$, which indicates that all of the residues in the peptide formed a helix, except for the residues located at the ends of the peptide. The average number of helices in one sequence was calculated according to Equation (23), and the results are shown in Figure 11. On the basis of the results shown in this figure, short peptides such as C17 and AK16 do not contain plural helices in the helix-coil transition region. Moreover, the average number of helices was nearly zero at higher $\mathrm{GuHCl}$ concentrations. With $\mathrm{C} 17$, the average number of helices was equal to 0.08 at a $\mathrm{GuHCl}$ concentration of $3.5 \mathrm{M}$. Thus, $92 \%$ of $\mathrm{C} 17$ molecules were not in the helix form under these conditions.

\section{Radius of gyration of $\mathrm{C} 17$}

$\mathrm{X}$-ray solution scattering was performed on $\mathrm{C} 17$, and the radius of gyration $\left(R_{\mathrm{g}}\right)$ was plotted against the $\mathrm{GuHCl}$ concentration (Figure 12). $R_{\mathrm{g}}=9.5 \AA\left(R_{\mathrm{H}}\right)$ at low $\mathrm{GuHCl}$ concentrations, and $\mathrm{Rg}$ $=12.8 \AA\left(R_{\mathrm{U}}\right)$ at high $\mathrm{GuHCl}$ concentrations. 

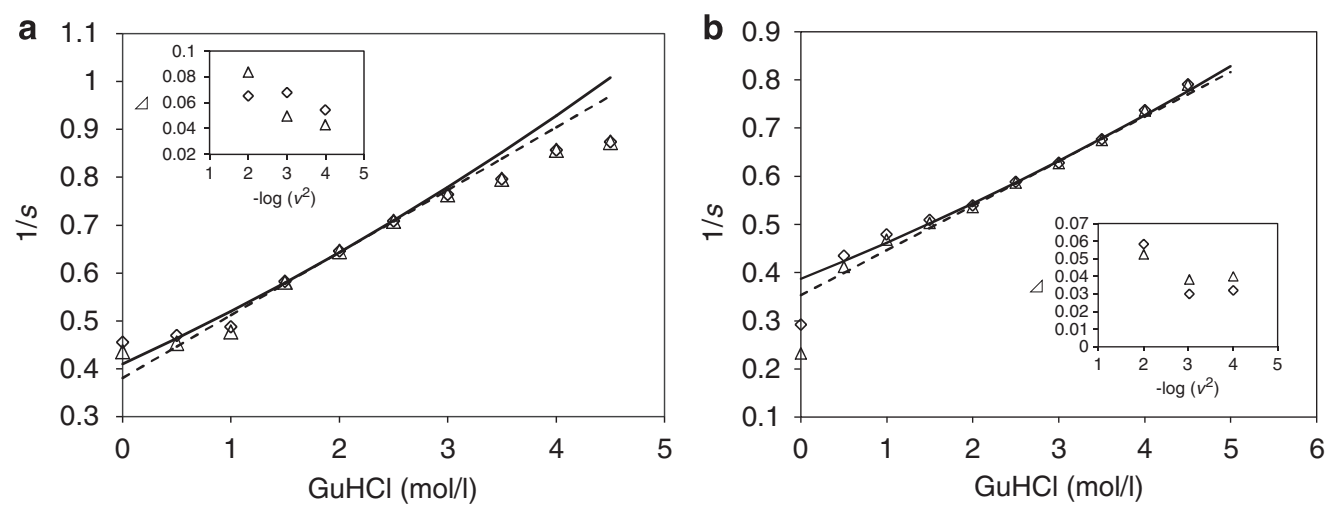

Figure $91 / s\left(v^{2}=10^{-4}\right)$ versus the guanidine hydrochloride $(\mathrm{GuHCl})$ concentration of the experimental data and fitted lines shown in Figure 8 . For the fitted lines, $1 / s$ was obtained from the fitted calculation. For the experimental data, $1 / s$ was calculated by relating $f_{\mathrm{H}}$ (obtained by Equation (38)) to $s$, according to Equation (21). (a) C17 and (b) AK16. $\Delta(p=1)$ and $\diamond(p=2)$ show the experimental results of $1 / s$. The broken line $(p=1)$ and solid line $(p=2)$ show the fitted lines of $1 / \mathrm{s}$. The insets show the relationship between $\Delta$ and $v^{2} . p=1(\triangle), p=2(\diamond)$.
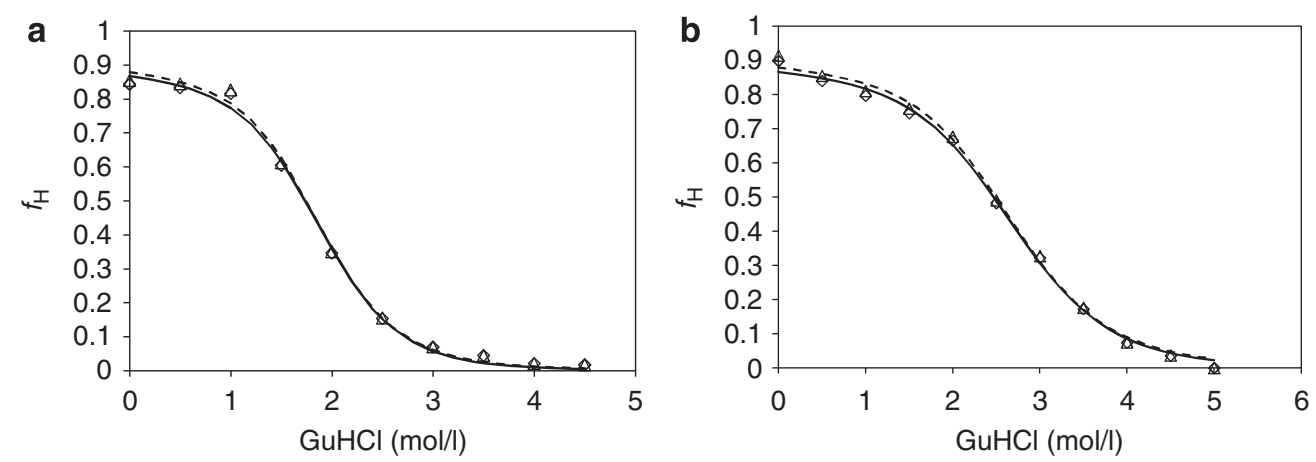

Figure 10 Calculated helix fractions $\left(f_{\mathrm{H}}\right)$ versus the guanidine hydrochloride $(\mathrm{GuHCl})$ concentration with experimentally obtained data. The fitted curves were obtained at $v^{2}=10^{-4}$ and $p=1$ and 2. (a) C17 and (b) AK16. $\Delta(p=1)$ and $\diamond(p=2)$ are the $f_{\mathrm{H}}$ values, which were calculated from the experimental data with the simulated parameters. The broken line $(p=1)$ and the solid line $(p=2)$ represent the calculated $f_{\mathrm{H}}$ values, which were obtained with the parameters described in the Results section.
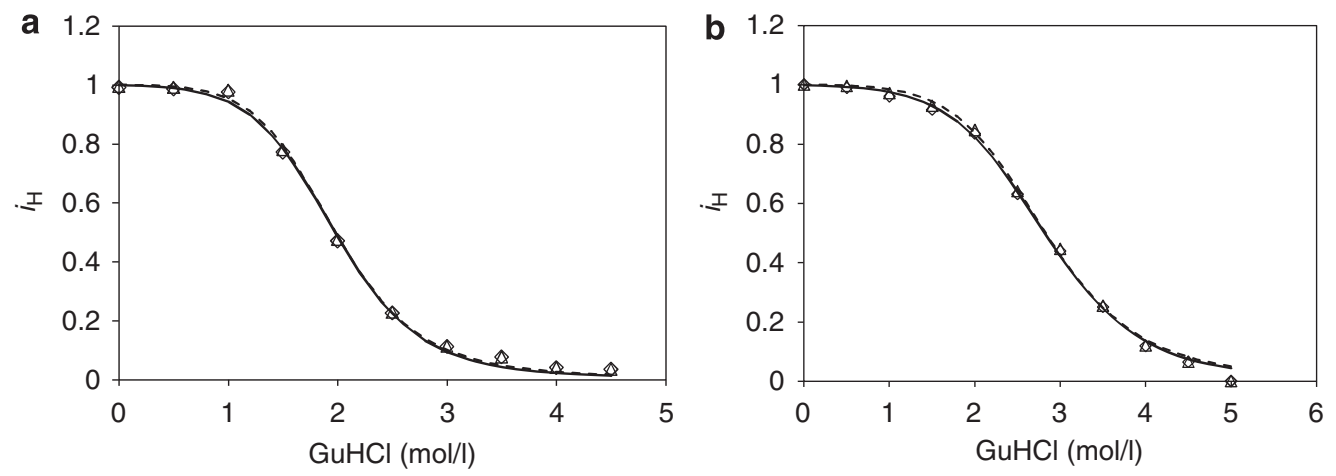

Figure 11 Calculated number of helices $\left(i_{H}\right)$ versus the guanidine hydrochloride $(\mathrm{GuHCl})$ concentration with experimentally obtained data. The fitted curves were obtained at $v^{2}=10^{-4}$ and $p=1$ and 2. (a) C17 and (b) AK16. $\Delta(p=1)$ and $\diamond(p=2)$ are the $i_{H}$ values obtained from the experimental data with the simulated parameters. The broken line $(p=1)$ and solid line $(p=2)$ show the calculated $i_{\mathrm{H}}$ values, which were obtained with the parameters described in the Results section.

$R_{\mathrm{g}}$ of the complete helix can be expressed by the following equation:

$$
\begin{aligned}
R_{g}= & \frac{1}{N} \sqrt{\sum_{i}\left(x_{i}-x_{g}\right)^{2}} \\
& =\frac{a}{N} \sqrt{\sum_{i}\left(i-\frac{1}{2}-\frac{N}{2}\right)^{2}}=\frac{a}{2} \sqrt{\frac{N^{2}-1}{3}}
\end{aligned}
$$

where $x_{i}$ is the coordinate of the $i$-th residue, $x_{\mathrm{g}}$ is the coordinate of the center of gyration and $a$ is the projection length along the axis of the residue. The results indicated that $a=1.9 \AA$, which is greater than the theoretical value of the $\alpha$-helix $(1.5 \AA) .{ }^{28}$ The calculation was conducted at $N=17$, and we assumed that all of the residues were present in the helical form.

$R_{\mathrm{g}}$ of the coiled state (Gaussian chain) can be expressed $\mathrm{as}^{29}$

$$
R_{g}=\sqrt{\frac{N}{6}} b
$$

where $b$ is the effective length per residue. These results indicated that $b=7.6 \AA$. However, if the excluded volume effect is considered, the 


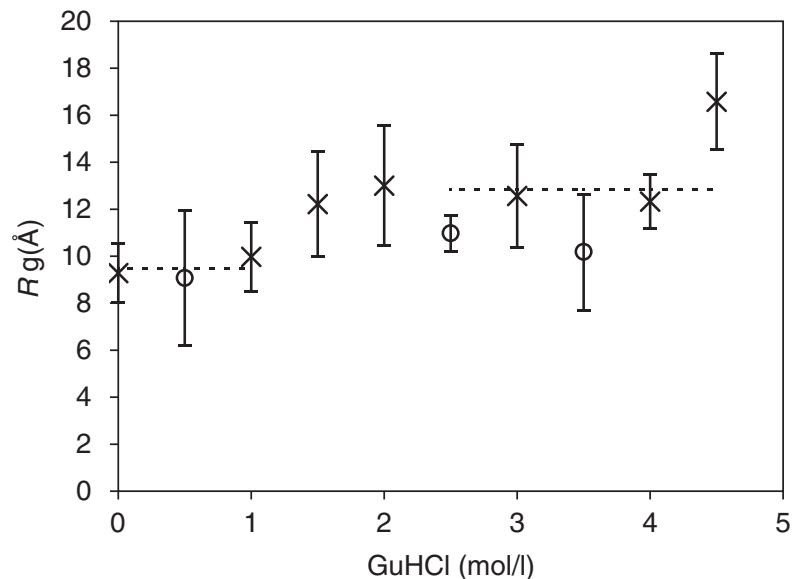

Figure 12 The dependence of $R_{\mathrm{g}}$ on the guanidine hydrochloride $(\mathrm{GuHCl})$ concentration. $x$ : three times the experimental average; $O$ : two times the experimental average The straight broken lines represent the average $R_{\mathrm{g}}$ of the helix $\left(0-1 \mathrm{~mol} \mathrm{I}^{-1}\right)$ and the coiled form $\left(2.5-4.5 \mathrm{~mol} \mathrm{I}^{-1}\right)$.

value of $b$ decreases. The transition from helix to coil was observed at denaturant concentrations of $1-2 \mathrm{moll}^{-1}$, which coincides well with the CD results.

\section{DISCUSSION}

The effects of the denaturant concentration on the helix-coil transition have been treated thermodynamically by Schellman. ${ }^{10,11}$ However, in the aforementioned derivation, intuitive treatment was implied. As shown in this study, statistical mechanics provide a satisfactory interpretation of the dependence of the helix-coil transition on the denaturant concentration, and detailed information was obtained.

As described in the Results section, the theoretical value of $\theta_{\mathrm{H} 0}$ for $\mathrm{C} 17$ and AK16 was $-29000\left(\mathrm{deg} \mathrm{cm}^{-2} \mathrm{dmol}^{-1}\right)$ and -22000 ( $\left.\operatorname{deg} \mathrm{cm}^{-2} \mathrm{dmol}^{-1}\right)$, respectively. According to Scholtz et al., ${ }^{12}$ the value of $\theta_{\mathrm{H} 0}$ for peptides is approximately -42500 $\left(\operatorname{deg} \mathrm{cm}^{-2} \mathrm{dmol}^{-1}\right)$. Initially, we attempted to fit the data to a $\theta_{\mathrm{H} 0}$ value of $-40000\left(\mathrm{deg} \mathrm{cm}^{-2} \mathrm{dmol}^{-1}\right)$; however, the simulation did not provide satisfactory results. Indeed, the value of $\theta$ for $\mathrm{C} 17$ is equal to $-36000\left(\mathrm{deg} \mathrm{cm}^{-2} \mathrm{dmol}^{-1}\right)$ in excess trifluoroethanol (unpublished data). In this study, the experimental value of $\theta$ in the absence of $\mathrm{GuHCl}$ was $-25000\left(\mathrm{deg} \mathrm{cm}{ }^{-2} \mathrm{dmol}^{-1}\right)$ for $\mathrm{C} 17$ and -20000 $\left(\mathrm{deg} \mathrm{cm}^{-2} \mathrm{dmol}^{-1}\right)$ for AK16. These values remained unchanged as the proportion of methanol increased. The saturated $\mathrm{CD}$ value was solvent dependent, which suggests that the $\alpha$-helix conformation may be dependent on the solvent.

The $s_{0}$ values reported by Scholtz et al. ${ }^{12}$ are lower than those obtained in this study. Nevertheless, when the value of $\theta_{\mathrm{H} 0}$ was set to $-42500\left(\mathrm{deg} \mathrm{cm}^{-2} \mathrm{dmol}^{-1}\right)$, the $s_{0}$ values were nearly identical to those obtained by Scholtz et al.; ${ }^{12}$ however, the fitting was poor.
1 Zimm, B. H. \& Bragg, J. K. Theory of the phase transition between helix and random coil in polypeptide chains. J. Chem. Phy. 31, 526-531 (1959).

2 Nagai, K. Configuration change of polypeptide molecules. J. Phys. Soc. Jpn. 15, 407-416 (1960).

3 Lifson, S. \& Roig, A. On the theory of helix-coil transition in polypeptides. J. Chem. Phys. 34, 1963-74 (1961).

4 Doig, A. J. Recent advances in helix-coil theory. Biophys. Chem. 101-102, 281-293 (2002).

5 Poland, D. \& Scheraga, H. A. Theory of Helix-Coil Transitions in Biopolymers, Academic Press, New York and London, 1970.

6 Tanford, C. Protein denaturation. Adv. Protein Chem. 23, 121-282 (1968).

7 Tanford, C. Protein denaturation C. Theoretical models for the mechanism of denaturation. Adv. Protein Chem. 24, 1-95 (1970).

8 Creighton, T. E. Proteins: Structure and Molecular Properties, (2nd edn.), (W.H. Freeman and Company, New York, 1993).

9 Fersht, A. Structure and Mechanism in Protein Science, (W. H. Freeman and Company, New York, 1998).

10 Schellman, J. A. Selective binding and solvent denaturation,. Biopolymers 26, 549-559 (1987).

11 Schellman, J. A. A simple model for solvation in mixed solvents: applications to the stabilization and destabilization of macromolecular structures. Biophys. Chem. 37, 121-140 (1990).

12 Scholtz, J. M., Barrick, D., York, E. J., Stewart, J. M. \& Baldwin, R. L. Urea unfolding of peptide helices as a model for interpreting protein unfolding. Proc. Natl. Acad. Sci. USA 92, 185-189 (1995).

13 De Gennes, P. G. Statistics of branching and hairpin helices for the dAT copolymer. Biopolymers 6, 715-729 (1968).

14 Doi, M. Helix-coil transition under external forces. Polymer J. 6, 222-229 (1974).

15 Kanô, $\mathrm{F}$. Theory of the transition between the intra $\beta$-structure and the random coil in polyamino chains. J. Phys. Soc. Jpn. 41, 219-227 (1976)

16 Kanô, F., Abe, I., Kamaya, H. \& Ueda, I. A fractal model for adsorption on activated carbon surfaces: langmuir and Freundlich adsorption isotherms. Surface Sci. 467, 131-138 (2000).

17 Doty, P. Configurations of biologically important macromolecules in solution. Rev. Mod. Phys. 31, 107-117 (1959).

18 Kanô, F. \& Doi, M. Dynamics of helix-coil transition and isotope exchange reaction. J. Phys. Soc. Jpn. 45, 1354-1359 (1978).

19 Bixon, M. \& Lifson, S. Solvent effect on the helix-coil transition in polypeptides. Biopolymers 4, 815-821 (1966).

20 Teramoto, A. Helix-coil transition of polypeptides. Seibutubutur 13, 149-163 (1973).

21 Amemiya, Y., Wakabayashi, K., Hamanaka, T., Wakabayashi, T. \& Hashizume, H. Design of a small-angle $\mathrm{X}$-ray diffractometer using synchrotron radiation at the photon factory. Nucl. Instrum. Methods 208, 471-477 (1983).

22 Amemiya, Y., Ito, K., Yagi, N., Asano, Y., Wakabayashi, K., Ueki, T. \& Endo, T. Largeaperture TV detector with a beryllium-windowed image intensifier for X-ray diffraction. Rev. Sci. Instrum. 66, 2290-2294 (1995).

23 Ito, K., Kamikubo, H., Arai, M., Kuwajima, K., Amemiya, Y. \& Endo, T. Calibration method for contrast reduction problem in the X-ray image-intensifier. Photon Factory Activity Rep. 18, 275 (2001).

24 Ito, K., Kamikubo, H., Yagi, N. \& Amemiya, Y. Calibration method and software for image distortion and non-uniformity in CCD-based X-ray detectors utilize X-ray image-intensifier. Jpn. J. Appl. Phys. 44 (Part 1), 8684-8691 (2005).

25 Pace, C. N. Determination and analysis of urea and guanidine hydrochloride $d$ enaturation curves. Methods Enzymol 131, 266-280 (1986).

26 Scholtz, J. M., Qian, H., York, E. J., Stewart, J. M. \& Baldwin, R. L. Parameters of helixcoil transition theory for alanine-based peptides of varying chain lengths in water. Biopolymers 31, 1463-1470 (1991).

27 Rohl, C. A., Scholtz, J. M., York, E. J., Stewart, J. M. \& Baldwin, R. L. Kinetics of amide proton exchange in helical peptides of varying chain lengths. Inter pretation by the Lifson-Roig equation. Biochemistry 31, 1263-1269 (1992).

28 Branden, C. \& Tooze, J. Introduction to Protein Structure (Garland, New York and London, 1991).

29 Saito, N. Koubunsi Butsurigaku (Shokabo, Tokyo, 1967). 